\title{
Probability-Based Location Aware Design and On-Demand Robotic Intrusion Detection System
}

\author{
Chia-How Lin and Kai-Tai Song, Member, IEEE
}

\begin{abstract}
For an on-demand robotic system, a location aware module provides location information of objects, users, and the mobile robot itself. This information supports various intelligent behaviors of a service robot in day-to-day scenarios. This paper presents a novel probability-based approach to building a location aware system. With this approach, the inconsistencies often seen in received signal strength indicator (RSSI) measurements are handled with a minimum of calibration. By taking off-line calibration measurement of a ZigBee sensor network, the inherent problem of signal uncertainty of to-be-localized nodes can be effectively resolved. The proposed RSSI-based algorithm allows flexible deployment of sensor nodes in various environments. The proposed algorithm has been verified in several typical environments and experiments show that the method outperforms existing algorithms. The location aware system has been integrated with an autonomous mobile robot to demonstrate the proposed on-demand robotic intruder detection system. In the experiments, three alarm sensors were employed to monitor abnormal conditions. If an intrusion was detected, the robot immediately moves to the location and transmits scene images to the user, allowing the user to respond to the situation in real time.
\end{abstract}

Index Terms-Autonomous navigation, location aware system, received signal strength indicator, security robot, sensor network.

\section{INTRODUCTION}

C ONVENTIONAL security systems have various limitations and shortcomings. For instance, a security agent employing conventional security systems has to dispatch security guards to respond to alarms. This creates a heavy burden for security agencies, especially considering a high portion of alarms are actually false alarms. Furthermore, it normally takes some time for the security guard to reach the alarm location to handle the situation. Critical time may have already been lost by the time the security guards arrive on the scene. One possible solution for such problems is to install a large number of security cameras throughout the guarded area to monitor any possible abnormal conditions. However, this solution may require high installation costs and pose privacy issues. The development of robotic security and monitoring

Manuscript received February 21, 2013; accepted May 28, 2013. Date of publication August 28, 2013; date of current version May 13, 2014. This work was supported by the National Science Council, Taiwan, under Grant NSC-96-2628-E-009-162-MY3. This paper was recommended by Associate Editor R. Roberts.

The authors are with the Institute of Electrical Control Engineering, National Chiao Tung University, Hsinchu 30010, Taiwan (e-mail: jotarun.ece87@nctu.edu.tw; ktsong@mail.nctu.edu.tw).

Color versions of one or more of the figures in this paper are available online at http://ieeexplore.ieee.org.

Digital Object Identifier 10.1109/TSMC.2013.2277691 systems for indoor environments, such as factories, offices, or home settings, has gained increasing attention in recent years [1]-[4]. An autonomous mobile robot provides sufficient mobility and flexibility to help security guards that lowers the relative cost as long as the demand for adequate security is satisfied.

For an on-demand robotic system, a locational aware module is required to provide location data of objects of interest, users and of the mobile robot itself [5]. This information supports various intelligent behaviors of a service robot in dayto-day scenarios. In the past decade, the production of various off-the-shelf devices for wireless sensor networks (WSNs) has progressed rapidly and such devices can support the use of ondemand robotic systems. WSNs are constructed using a series of sensor nodes deployed throughout the monitored environment. These sensor nodes are connected and communicate with each other over a wireless network such as wireless LAN, Bluetooth, ultrawide band, or ZigBee. This allows the use of free-ranging autonomous service robots that operate in human centered environments to assist people. Because the robot has limited onboard sensing and computing abilities, integrating service robots with a WSN is desirable to enable robots to be used for more practical applications. A WSN can serve as a low-cost, distributed, and easily deployed monitoring system that extends the limited sensing capability of the robots.

Unlike a stand-alone security robot, which is normally equipped with a variety of sensors but still has only a limited sensing range, on-demand security robots can obtain thorough sensory information of the guarded area and make it available on line. When integrated with the WSN, a security robot can acquire information from the entire sensed environment in real time. To enhance the prompt response of security robots to an intrusion situation, many researchers have studied the application of WSN and mobile robot techniques for use in security and monitoring [6], [7]. WSN-based localization can estimate the location of a mobile node with an initially unknown position in a sensor network using available $a$ priori knowledge of positions of a few specific reference nodes in the network. However, most of these designs require overly complex and impractical installation procedures and suffer from problems with signal calibration/training. For example, some wireless sensor modules have to be placed in restricted locations such as on a ceiling [3].

Localization algorithms should meet the requirements of various hardware configurations such as signal transmission, power requirements, and computational complexity. These 
factors allow us to divide various approaches into three main categories [8]: range-free, range-based, and fingerprinting. Range-free localization [9] is based on the connectivity of the network. It does not require any special hardware and the content of the messages is received through simple operations. Range-based localization, on the other hand, estimates the distance between nodes using certain ranging techniques. The distance information can then be used to locate the position of unknown sensor nodes. Most range-based localization algorithms adopt the received signal strength indicator (RSSI) technique to estimate the distance based on the strength and path loss model of the signal that was received. After RSSI is used to estimate the distance, the second phase of localization performs computations based on the relative distance between nodes. Many strategies exist for location estimation such as multilateration [10] and min-max [11]. Unfortunately, indoor radio channels are unpredictable, because reflections of the signal against walls or ceiling may result in severe multipath interference at the receiving antenna. A straightforward way to overcome the inaccuracy of RSSI is to use enhanced or additional radio hardware, such as multiple directional antennas [12]. However, the devices used in these solutions generally demand more energy and are much more expensive to deploy.

The fingerprinting approach or location pattern matching techniques [13], [14] are based on the concept of identifying a specified position by relying on RSSI data received from nearby nodes. This approach uses two phases, a training phase, and an estimation phase. In the training phase, the RSSI is measured at grid points in the area of interest. This information is used to estimate the propagation model parameters, which are employed later in the estimation phase. The accuracy of the calibration procedure depends on the number of points in the grid and the number of measures taken per point. Since the fingerprinting approach is basically a pattern matching and generalization technique, many researchers have applied state-of-the-art intelligent-computation approaches to resolve this problem. Oliveira et al. [15] combine the RSSI technique and the link quality indicator (LQI) using fuzzy logic and transferable belief model (TBM). Their results indicate that this type of combination metrics can refine the estimated distances. Gogolak et al. [16] preprocess the RSSI values to obtain the mean, median, and standard deviation of the data and uses these processed data to train a neural network in order to increase the accuracy of the localization system. Fang and Lin [17] proposed the discriminant-adaptive neural network (DANN), which extracts useful information into discriminative components (DCs) for neural network learning. The nonlinear relationship between RSSI and the object's position is then accurately constructed by incrementally inserting the DCs and recursively updating the weights in the network until no further improvement is required. In general, these techniques yield improved performance when the number of nodes is relatively large. However, they are very time consuming as the process requires exhaustive data collection, which is a practical barrier to its wider adoption.

Due to the long calibration time required for fingerprinting approaches, range-based methods are more suitable in applications where fast deployment is necessary. The most

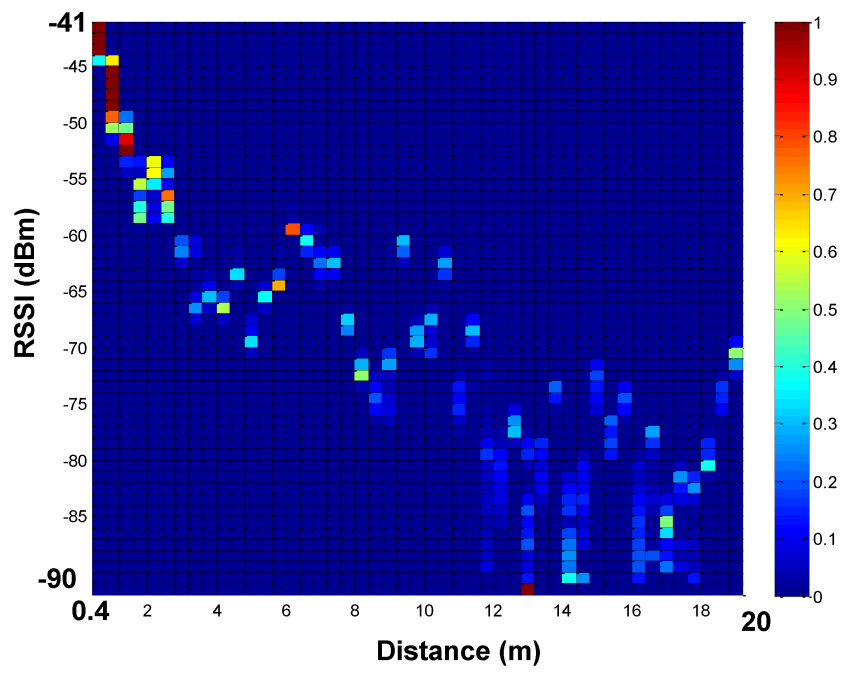

Fig. 1. Example of RSSI irregularity.

challenging problem is how to overcome the irregularity of range estimation using RSSI. Ramadurai and Sichitiu [18] proposed to merge all the data collected and estimated the probability distribution of WiFi radio frequency (RF) signal strength as a normal distribution function of distance. While their results indicate that the actual position of a signal node is well bounded by the estimated position obtained despite ranging inaccuracies, their method is not suitable for an indoor environment because of the presence of multipath fading. With lower power, stronger shadowing and fading effects, it is even more difficult to find a proper distribution for ZigBee-based systems. Fig. 1 shows an example of the relationship between RSSI and distance using ZigBee modules. As the figure shows, the relationship between RSSI and distance is quite irregular, and a perfect mathematical model that adequately describes this type of distribution is difficult to find. In [19], a thorough free space loss model of RF signal is proposed. This model considers the irregularity seen in RSSI and the parameters of the RF hardware (antenna gain and efficiency, transmit power, etc.). However, this subtle model assumes that only the direct signal reaches the antenna with the RSSI value, and may still fail in real-life situations. Similar observations can also be found in many other attempts [20]-[22]. When the model fails to accurately describe the relationship between RSSI and distance, overall accuracy will decrease dramatically. Lee et al. [22] used both unscented Kalman filtering (UKF) and particle filtering (PF) to track a target tag with RSSI. The UKF assumes near linearity and unimodal Gaussians in the system while the PF does not. Their results show that the assumptions of UKF are very fragile and easily violated. Furthermore, while the tracking method itself is verified, several sets of parameters for the RSSI model for different parts of the area need to be determined beforehand, to overcome the irregularity.

In summary, the currently available location-aware solutions, whether off-the-shelf or state-of-the-art approaches, suffer from the requirement of prior parameter training and retraining in different application areas. It is difficult or even impossible to build up a location aware system in a totally 
new environment. This motivated us to develop a new localization method that can provide a straightforward and practical solution for sensor-node calibration and provide WSN localization with acceptable accuracy. The proposed method requires no extra hardware. Most importantly, calibration is only needed once and provides adequate robustness in different environments.

The proposed location aware system has been integrated and tested with an intruder detection system that features a ZigBee WSN and a mobile robot. If any intruders or abnormal conditions are detected, the state and location of this alert will be transmitted to the security robot and monitoring center on the WSN. The robot can navigate autonomously to the alarm location using the probability-based localization system. After the robot arrives on the scene, the onboard camera will transmit real-time images to the user via both $\mathrm{WiFi}$ and $3 \mathrm{G}$ networks. The security guards and end-users can therefore easily determine the exact situation in real time.

The remainder of this paper is organized as follows. Section II presents the proposed probabilistic localization method. Section III describes the system design and implementation of the on-demand intrusion detection robotic system. Section IV presents the results of the experiment describing and discussing both the performance evaluation of the localization system and validation of the intrusion detection technique. Section V summarizes the contributions of this paper.

\section{Proposed Probability-Based ZigBeE LOCALIZATION METHOD}

To overcome limitations posed by uncertainties of RSSI, we suggest a novel probability-based approach to estimating location by modeling the RSSI versus distance relationship with discrete probability density functions. This approach aims to provide a straightforward method of describing different distributions without losing generality. While most current approaches use mathematical equations to model the RSSI versus distance relationship, the proposed method adopts the original RSSI versus distance data to construct its own model. Furthermore, instead of using trilateration or fingerprint techniques, the location is estimated by accumulating a probability histogram observed from several reference nodes on a local probability map. This approach will be shown to have better tolerance against fluctuations and inconsistencies inherent with the RF signal. It is also flexible because it uses the available number of the RSSI measurements and the deployment of ZigBee nodes.

We first assume that the locations of all reference nodes are already known and the RSSI between each pair of nodes can be received. Fig. 2 shows a flow chart of the algorithm. It contains a calibration phase that models the RSSI versus distance relationship and a localization phase that estimates the location using a filtered 2-D probability map as follows.

\section{A. Modeling the RSSI versus Distance Relationship}

The calibration phase estimates the distance between the nodes based on the RSSI measurement. Instead of using a

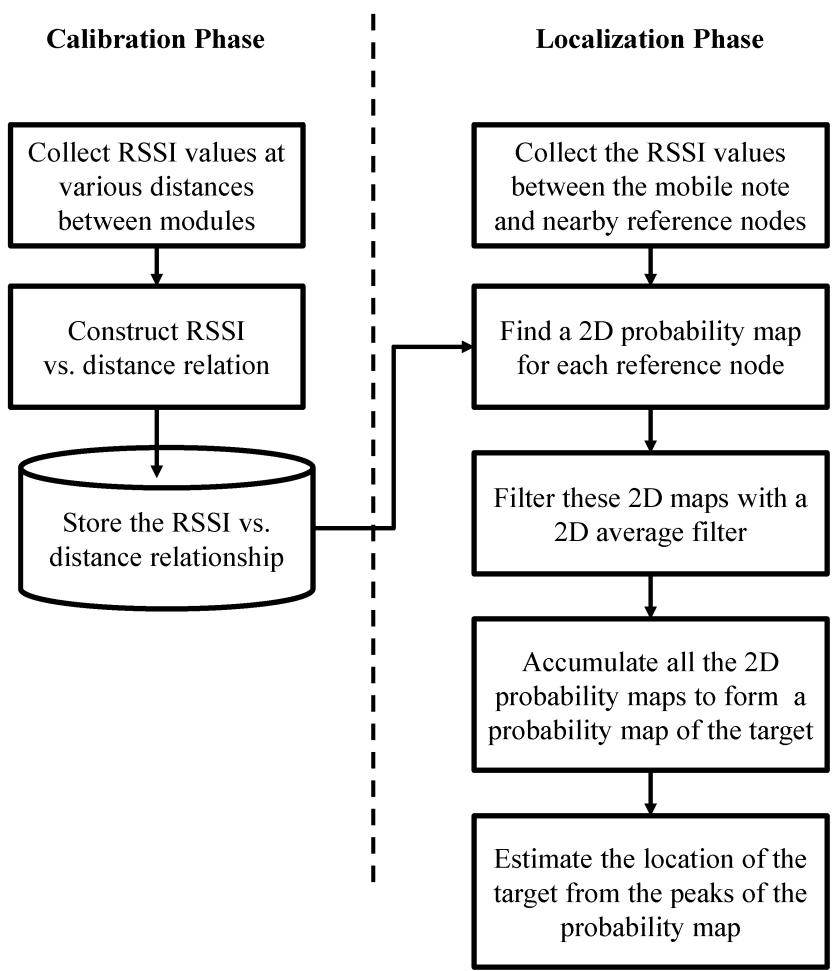

Fig. 2. Flow chart of the proposed localization algorithm.

mathematical path-loss model, the RSSI versus distance relationship is collected and modeled with a series of probability histograms, which records different distances measured under a fixed RSSI. The histogram therefore represents the discrete probability density function (pdf) of a given RSSI value. Let $R$ and $D$ denote the random variables of the RSSI reading and distance between the nodes (in meters), respectively. Assume the model is tested from distance 0 to $L$ meters at intervals of $q$ meters. The pdf of the RSSI value equal to $r$ can be defined as

$$
\mathrm{P}(D \mid R=\mathrm{r})=\sum_{i=0}^{N-1} \mathrm{P}_{D}\left(\mathrm{~d}_{\mathrm{i}} \leq D \leq \mathrm{d}_{\mathrm{i}+1} \mid R=\mathrm{r}\right)=\sum_{i=0}^{N-1} h_{i}
$$

where $d_{i}$ represents the distance of the $i$ th interval, $N=L / q$ is the total bins in the histogram and $h_{i}$ is the value of each bin. Fig. 3 shows an example of the probability histogram when RSSI $=-88 \mathrm{dBm}$. This model can easily be implemented onboard the sensor node since it only needs a single look-up table. Clearly, the number of the bins in each histogram limits the resolution of the estimation of distance. This is, however, not critical since the RSSI value is also discrete in practice.

\section{B. 2-D Probability Map}

In the next step, the location of the mobile node is estimated using the RSSI values measured from several nearby reference nodes. The concept is to apply the 2-D multilateration method with the estimated pdfs. A trilateration method determines the location by finding the intersections of circles given from: 1) the centers of the reference nodes and 2) distance between the mobile node and several reference nodes (more than three). In the proposed algorithm, however, a mobile node 


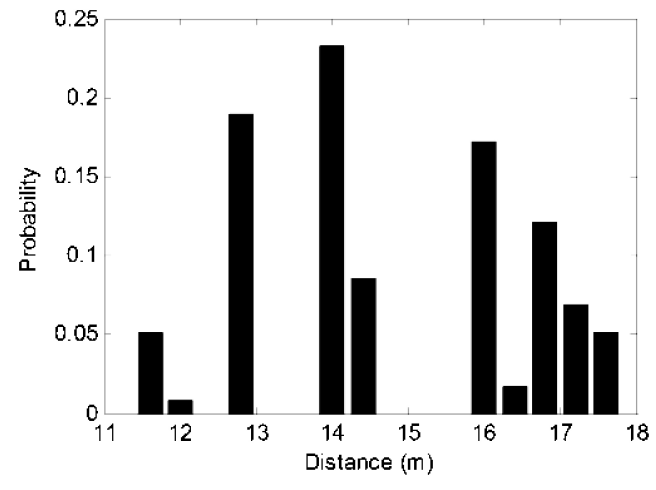

Fig. 3. Probability histogram of distance for RSSI $=-88 \mathrm{dBm}$.

may obtain several possible distance measurements from each reference node. The next step is to find the intersection sets of distance measurements with the largest probability from several reference nodes.

To simplify the calculations, the proposed algorithm estimates the 2-D probability distribution directly on the 2-D map quantized in grids of a fix size of $\mathrm{K} \times \mathrm{K}$. Specifically, grids of the size $\mathrm{N} \times \mathrm{M}$ are spanned over the localization area, defined by $(x, y), 1 \leq x \leq \mathrm{N}$, and $1 \leq y \leq \mathrm{M}$. The next step is to derive the location of the mobile node $\mathbf{x}_{\mathrm{m}}=\left(x_{\mathrm{m}}, y_{\mathrm{m}}\right)$ from an RSSI vector $\mathbf{R}=\left(R_{1}, R_{2} \ldots, R_{i}, \ldots R_{K}\right)$, containing the RSSI values received from $K$ reference nodes with known positions $\boldsymbol{x}_{r, i}$, $1 \leq i \leq K$. For each reference node $\boldsymbol{x}_{r, i}$ with an RSSI value $r$ measured at the mobile node, the 2-D pdf $\mathrm{P}(x, y, i)$ of the mobile node at position $(x, y)$ can be given as

$$
\begin{aligned}
\mathrm{P}(x, y, i) & =\sum_{y=1}^{M} \sum_{x=1}^{\mathrm{N}} \mathrm{P}_{X, Y}\left(\mathbf{x}_{m}=(x, y) \mid \mathrm{R}_{\mathrm{i}}=\mathrm{r}\right) \\
& =\sum_{y=1}^{M} \sum_{x=1}^{\mathrm{N}} \mathrm{P}\left(D=\left\|\left(\mathbf{x}_{r, i}-(x, y)\right)\right\| \mid \mathrm{R}_{\mathrm{i}}=\mathrm{r}\right) .
\end{aligned}
$$

Since the RSSI versus distance model is known and modeled as a 1-D pdf, the 2-D probability distribution is equivalent to a repeat of the 1-D pdf $\mathrm{P}(\mathrm{D} \mid \mathrm{R}=\mathrm{r})$ in (1) around the coordinates of the reference node from 0 to $360^{\circ}$. Fig. 4 shows a graphical example of the 2-D pdf with one mobile node and one reference node at $(0,0)$. The 2-D pdf indicates the probability of the mobile node by means of a 2-D probability map.

\section{Filtered 2-D Probability Map}

While the localization method described in the previous section works under many circumstances, some practical issues still need to be resolved. Among them, the most important problem is the gap in the histogram created by the inconsistency of the RSSI values. For instance, in Fig. 3 (when RSSI $=-88 \mathrm{dBm}$ ), the probability of the distance $=15 \mathrm{~m}$ is zero, which is unlikely in reality, because of the irregular nature of the radio signal. To realize the goal that the model only needs to be built once, we need to enhance the proposed model to tolerate these circumstances. Furthermore, for some WSN applications such as searching, it would also be beneficial if the proposed method can provide alternative locations when

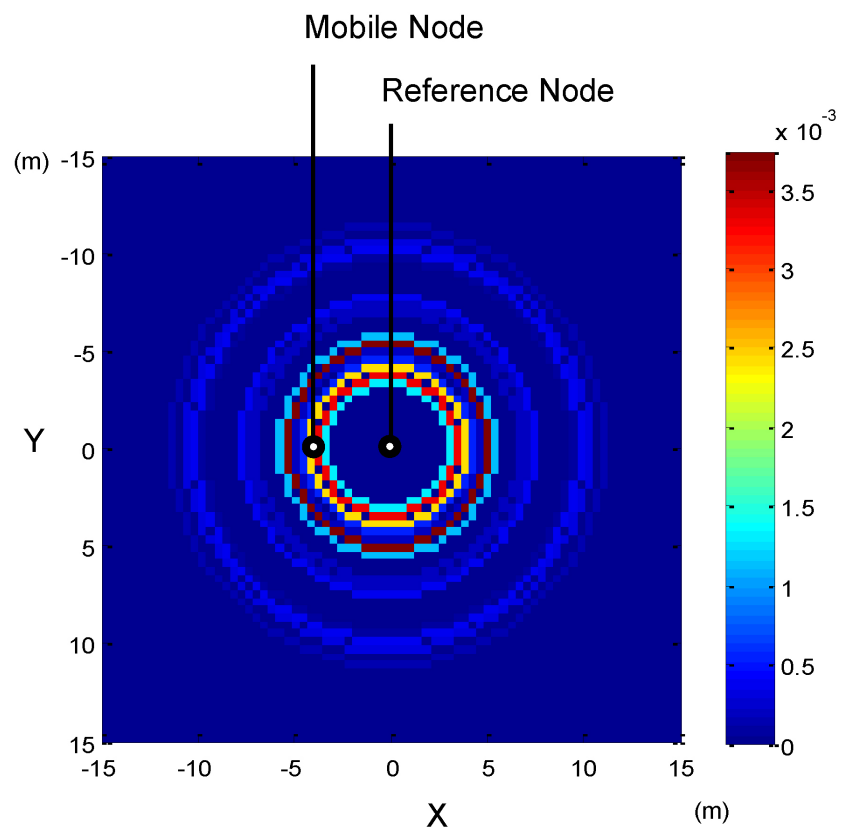

Fig. 4. 2-D pdf of a fixed RSSI reading between a reference node and a mobile node.

it fails on the first try. In order to resolve these issues, we treat the 2-D probability map as an image and apply a 2-D circular averaging filter $\mathrm{H}(x, y)$ with radius $r$ to it. The idea is to smoothen the map by filling the gaps and avoid any zero probability on the map by taking probabilities of nearby areas into account. The filter is defined as

$$
H(x, y)=\frac{1}{r^{2} \pi} \cdot \begin{cases}1, & \text { if } x^{2}+y^{2} \leq r^{2} \\ 0, & \text { otherwise }\end{cases}
$$

where the value of $r$ is adjusted according to the variance of the RSSI values. The filtered map is thus stated as

$$
\hat{P}(x, y, i)=P(x, y, i) * H(x, y) .
$$

As a result, the filtered map provides a relatively more robust result than the raw map (refer to the experiment section). The filtered map also provides other possible locations for the robot or human to search for the target, if they failed to find the target at the location with the highest probability.

\section{Location Estimation}

Finally, the location of the current mobile node will be estimated by accumulating all the 2-D probability maps obtained from reference nodes such that

$$
P(x, y)=\sum_{i=1}^{K} \hat{P}(x, y, i) .
$$

The summation of each grid $P(x, y)$ is analogous to the convolution of all the individual distributions. Fig. 5 shows an example of how maps obtained from different reference nodes are accumulated into the final map. As a result, the mobile node is located by the peak value of the probability map, even though the upper-right map suggests a false result. However, because of the natural irregularities of the RSSI, the 


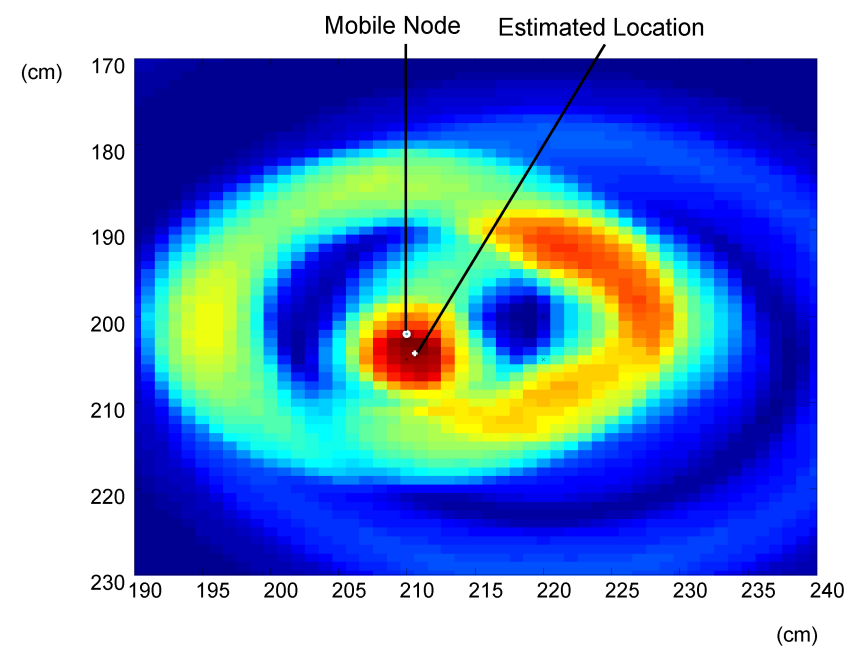

Fig. 5. Example of how filtered 2-D maps obtained from different reference nodes are combined into the final map.

results may lead to a map with several peaks. The location of the mobile node will not be determined by one of the peaks under those circumstances. Instead, the propose algorithm determines the location by estimating the geometric center of all the peaks such that

$$
\mathbf{x}_{m}^{*}=\frac{\sum_{p=1}^{\mathrm{P}} \mathbf{x}_{p}}{\mathrm{P}}
$$

where $\boldsymbol{x}_{p}$ denotes location of the $p^{\text {th }}$ peak on the 2-D pdf, $1 \leq p \leq P$. Note that the multilateration method can now be treated as a special case of the proposed algorithm, where the pdf of each reference node is an impulse function.

\section{SySTEM DESIGN AND IMPLEMENTATION}

ZigBee is a popular ad hoc network based on the standard IEEE 802.15.4. ZigBee Alliance [23] defines the specification of ZigBee for networks and higher layers. The ZigBee standard has many advantages for real-time positioning systems in terms of battery life and network size. It is widely used in low data rates, low power, and cost-effective wirelessly networked products. Fig. 6 shows a typical implementation of the developed localization system for robotic intrusion detection [24]. In this system, the mobile robot is equipped with two driving wheels (differential drive) and two casters for balance. Sixteen ultrasonic range finders and eight infrared proximity sensors were installed onboard the robot for obstacle avoidance.

\section{A. Intrusion Detection Sensors}

We designed several useful sensor modules for intrusion detection to verify the effectiveness of the proposed algorithm. Fig. 7 shows such an intruder detection sensor module. It consists of an 8-bit microcontroller Atmega128L (Atmel Corp.), a ZigBee chip CC2420 (Texas Instruments), and an onboard Freescale MMA7260QT triaxial accelerometer (Freescale Semiconductors). Two other sensor modules, a pyro sensor and a microphone sensor, were also designed and

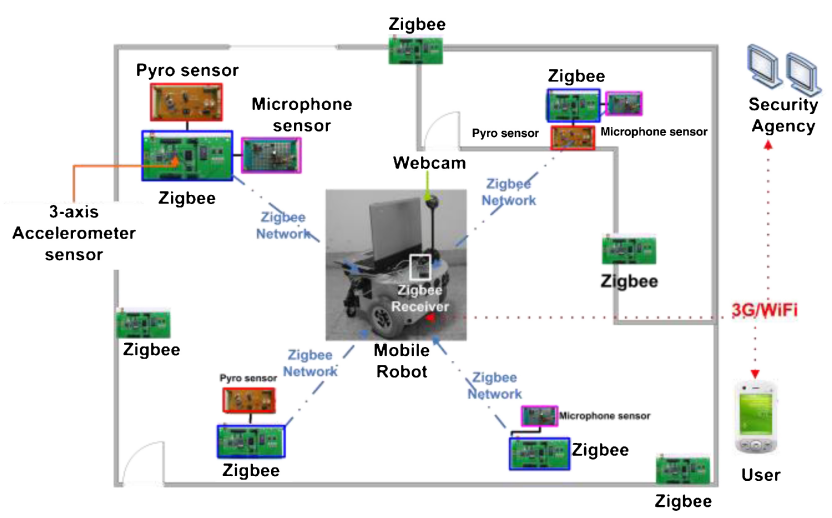

Fig. 6. Hardware arrangement of an intruder detection system.

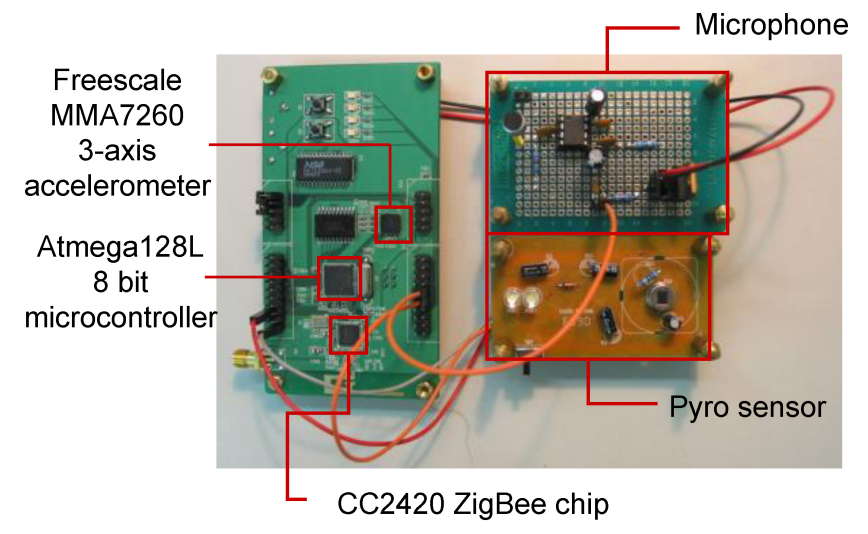

(a)

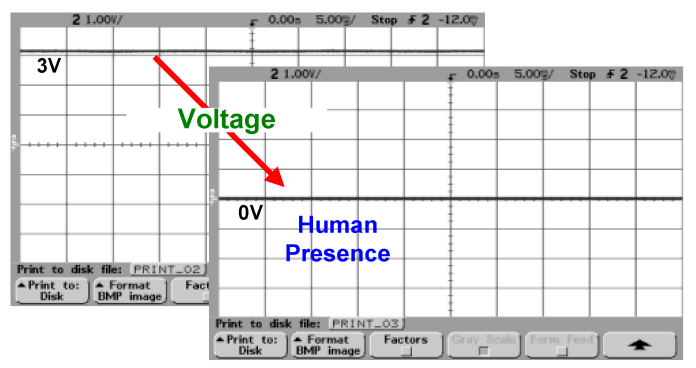

(b)

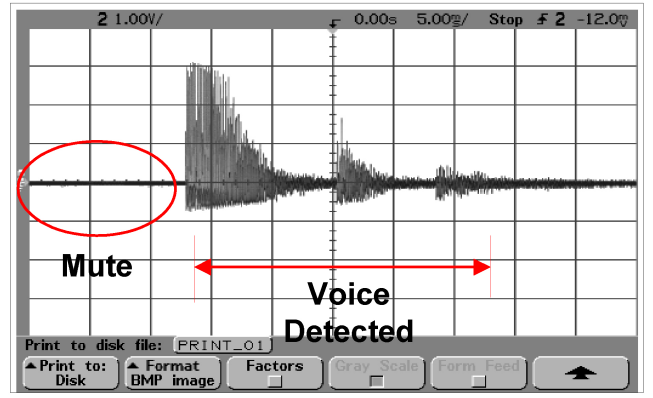

(c)

Fig. 7. Intruder detection module. (a) Hardware of the module. (b) Signal of the pyro sensor. (c) Signal of the microphone.

integrated into the experiments. The details of these sensor modules are described as follows.

1) Triaxial accelerometer: The triaxial accelerometer is applied to detect vibrations. Individual magnitudes of the dynamic acceleration from $x, y$, and $z$ axes are first isolated. 
The summation of these magnitudes can thus be used to detect abnormal vibrations during an intrusion or collision.

2) Pyro sensor: We adopted off-the-shelf pyro-electric sensor components. The pyro sensor can detect the presence of humans in a certain area by a change in the voltage level of sensor output. As Fig. 7(b) shows, if the voltage level is close to zero, the sensor will be triggered.

3) Microphone: A simple microphone was integrated into the ZigBee module for abnormal sound detection. The rising edge of the filtered microphone signal will trigger the interrupt of the chip and can be used to determine loud sounds [Fig. 7(c)]. The trigger level is set at $+2.01 \mathrm{~V}$. A loud sound can be caused by a falling object resulting from an intrusion or the breaking of a window.

If any sensor is triggered, a message including the current time, the ID of the triggered sensor, and the location of the node will be sent to the robot. Since the robot may be far from the location, an adaptive route selection algorithm is applied to send the message by forwarding data packets to the robot via selected sensor nodes [25].

\section{B. Mobile Robot Localization}

Several reference nodes were deployed beforehand in the environment to localize the robot using the proposed method. Their positions were stored in a database on the robot's onboard computer. Two kinds of messages are defined for the ZigBee WSN: the measurement-demand and signal-report. The measurement-demand message is used for a mobile node to request the RSSI measured from reference nodes. This message is broadcasted to all the nodes that are able to receive it. Furthermore, a counter is included in this message to keep the track of various measurement demands. The signal-report message is used by the reference nodes to report the measured RSSI values. In summary, location estimation is performed using the messages and the following process.

1) A measurement-demand message is broadcasted to sensor nodes from a mobile node.

2) Each sensor node measures RSSI at the time it receives the packet. Subsequently, it transmits the RSSI, counter number, and the mobile node ID to the robot.

3) The robot collects all the data, separates each by the ID and counter. If three or more RSSI values with the same counter are observed, the location of the robot can be estimated and updated using the proposed algorithm. In the current implementation, the entire procedure can be accomplished within $600 \mathrm{~ms}$.

\section{Autonomous Navigation System}

Autonomous navigation in a dynamic changing environment is essential for a robot patrolling from one place to another. In the proposed system, this task is achieved by a behavior-fusion approach adopted from [26]. Fig. 8 illustrates the architecture of navigation system using behavior fusion. We designed three primitive behaviors for autonomous navigation in an indoor environment. These behaviors are goal seeking behavior, wall following behavior, and obstacle avoidance behavior. Goal seeking behavior is treated as an attempt to move toward

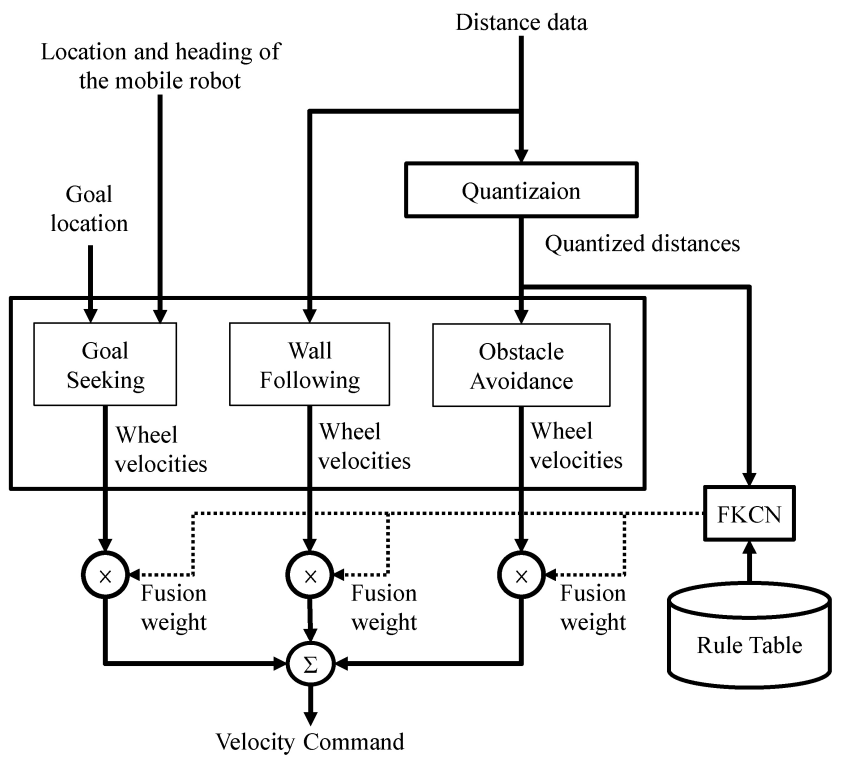

Fig. 8. System architecture of autonomous navigation using behavior fusion design.

the direction of the target. Wall following behavior is defined as maintaining the same distance from the nearest wall. The obstacle avoidance behavior is designed so that the robot moves in a direction away from any obstacles. These behaviors will each output a set of desired wheel velocities.

The concept of pattern recognition was adopted to fuse the output of navigation behaviors. The pattern recognition technique was used to map the environmental configuration, obtained from ultrasonic and infrared sensors onboard the robot, to the fusion weights of three navigation behaviors. The concept can be expressed as: when the environment configuration of surroundings is similar, the fusion weights of navigation behaviors should be similar. In this paper, the fuzzy Kohonen clustering network (FKCN) is applied to map the environmental patterns to fusion weights [26]. In this design, 10 prototype patterns defined by the quantized distances to objects around the robot were set manually to represent typical environmental configurations for indoor navigation. Suitable fusion weights for each navigation behavior corresponding to these patterns were assigned in the rule table of FKCN. When the robot navigates through the environment, the sensors will obtain current range data around the robot. Then the FKCN works to generate proper fusion weights for each navigation behavior, corresponding to the immediate environmental sensory data pattern. As a result, the mobile robot can navigate to the desired location without colliding into any objects in the environment. In this application, the positions of both the robot and the goal will be determined by the location aware system.

\section{EXPERIMENTAL RESULTS}

\section{A. Localization Robustness Test}

The experiment aims to test if the proposed method can handle ZigBee localization in different environments more 
TABLE I

CONFIGURATIONS OF THE TEST ENVIRONMENTS

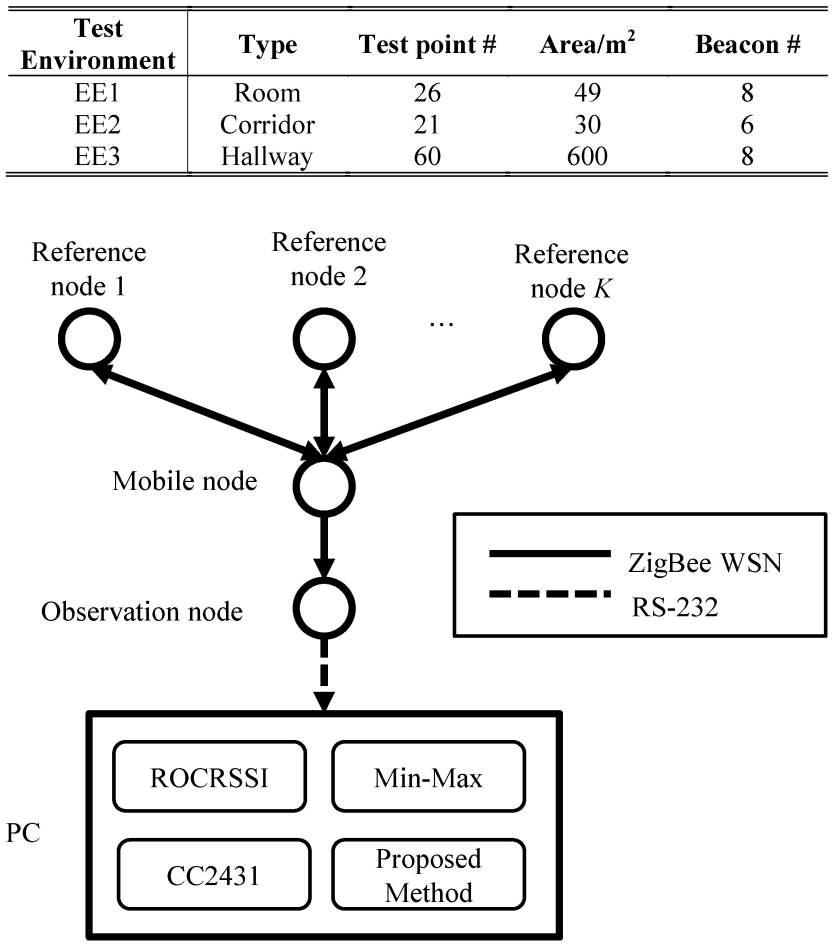

Fig. 9. Data flow of the ZigBee localization experiments.

robustly than the other methods. Three localization methods: min-max, ROCRSSI, and CC2431 are compared with the proposed method in this experiment. Min-max is a widelyadopted range-based localization method [11]. The technique known as ring overlapping based on comparison of received signal strength indication (ROCRSSI) is a commonly used range-free approach [9] to localization. The CC2431 location engine is embedded in a Texas Instruments CC2431 systemon-chip (Soc) solution for ZigBee/IEEE 802.15.4 wireless sensor networks. CC2431 implements Motorola's IEEE 802.15.4 radio-location solution exploiting a maximum likelihood estimation algorithm [23]. To compare the CC2431 location engine with the proposed method, we have adopted our modules with CC2431 chips to perform the experiment.

Fig. 9 shows the data flow of the experimental system. RSSI values of reference nodes were first estimated by the mobile node. The values were then sent to the observation node and finally transmitted to the host PC via a serial link. The collected RSSI values were then estimated with different algorithms and compared with ground truth. Three experiments have been performed in three different types of environments. Table I shows the configurations of these environments. The localization result using raw data as well as the filtered 2-D map were individually tested in the experiment to further investigate the effectiveness of the proposed method. Figs. 10-12 provide photos and the locations of beacons in these environments. During the calibration measurements of the proposed method, RSSI values at various distances in several different environments of the lab building were recorded.

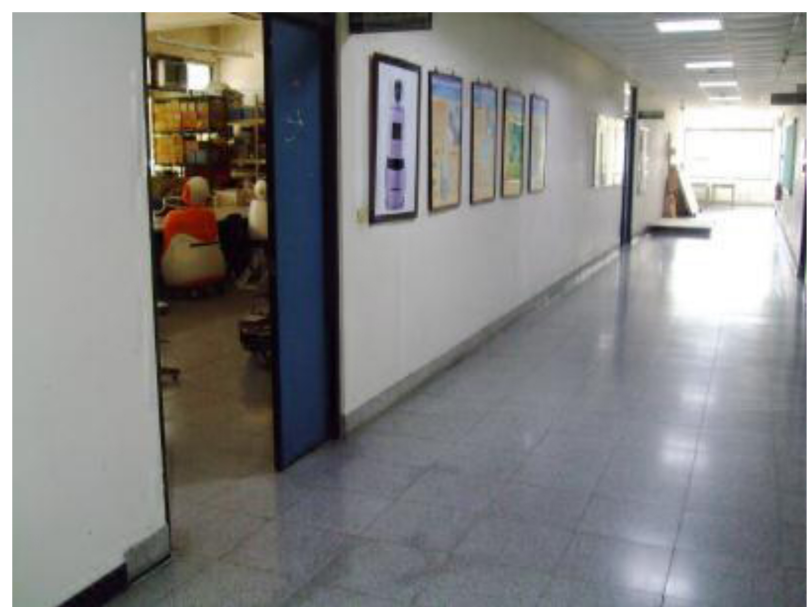

(a)

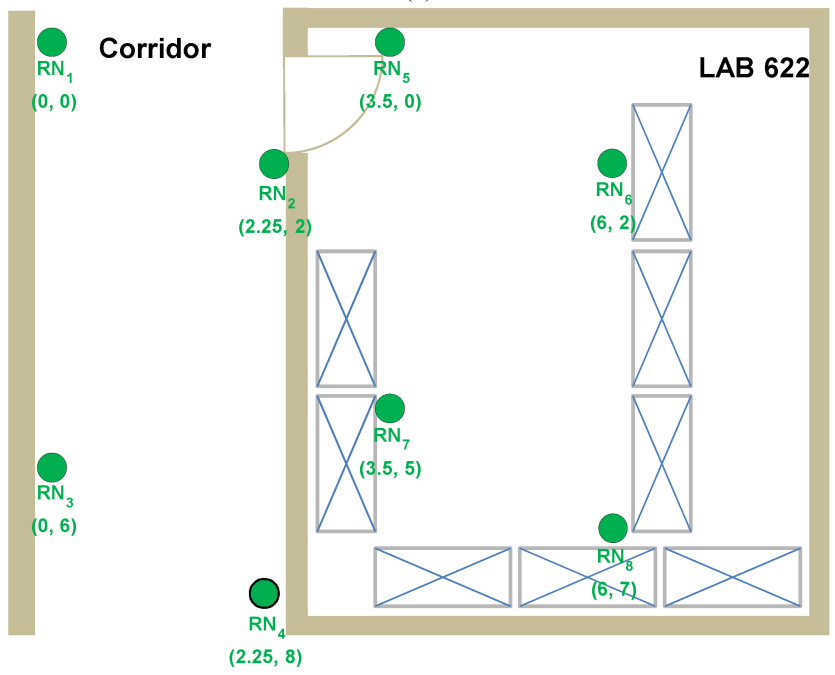

(b)

Fig. 10. (a) Experimental environment 1 (EE1). Lab 622 and corridor. (b) Location of deployed ZigBee beacons in the experiment (filled circles).

In the measurement, the RSSI values between nodes were collected by moving a mobile node away from a reference node manually along a direction from a distance of $0-10.2 \mathrm{~m}$ with an interval of $0.3 \mathrm{~m}$. The goal is to collect RSSI data under different circumstances. The calibrations of the other methods were performed only in experiment environment 1 (EE1). In the experiments, every ZigBee module was fully charged before the experiment, since the power of ZigBee sensor nodes affects RSSI and hence the location prediction.

Table II shows the overall localization results of EE1. The results show practically no difference in the localization error between the proposed method and the CC2431 localization engine. Both results outperform the Min-Max and ROCRSSI methods, which is similar to the results reported in [8]. Notice that the parameters of the signal propagation model used in the CC2431 localization engine are given to maximize the accuracy of the experiment, while our approach uses the sensor model established beforehand from other calibration places.

In experiment environments 2 (EE2) and 3 (EE3), the same RSSI models and parameters were applied as were used in EE1. Tables III and IV show the overall localization 


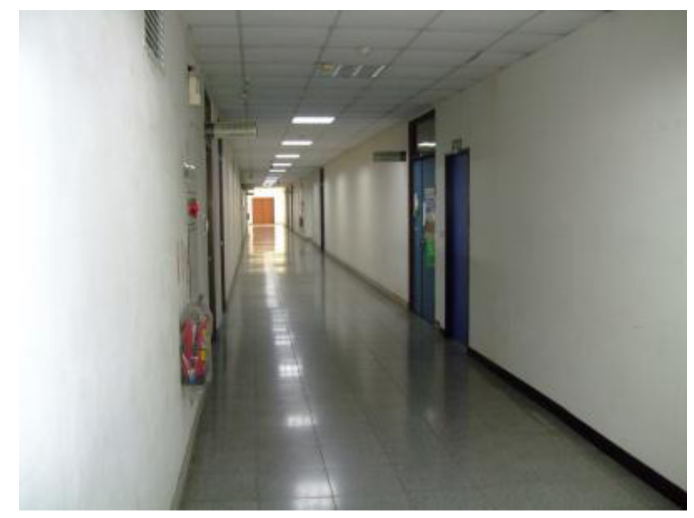

(a)

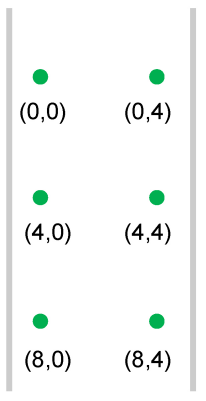

(b)

Fig. 11. (a) Experimental environment 2 (EE2). Corridor of lab building. (b) Location of deployed ZigBee beacons in the experiment (filled circles).

TABLE II

ESTIMATION ERROR COMPARISON: EE1

\begin{tabular}{c|ccc}
\hline \hline Method & $\begin{array}{c}\text { Average } \\
\text { Error }(\mathbf{m})\end{array}$ & $\begin{array}{c}\text { Max Error } \\
(\mathbf{m})\end{array}$ & $\begin{array}{c}\text { Standard } \\
\text { Deviation }\end{array}$ \\
\hline Min-Max & 2.29 & 3.4 & 0.77 \\
ROCRSSI & 2.59 & 3.5 & 1.11 \\
CC2431 & 0.91 & 3.25 & 0.64 \\
$\begin{array}{c}\text { Proposed } \\
\text { (2D Map) }\end{array}$ & 1.29 & 3.3 & 0.82 \\
$\begin{array}{c}\text { Proposed } \\
\text { (with Filtered Map) }\end{array}$ & 1.12 & 3.5 & 0.89 \\
\hline \hline
\end{tabular}

error of the experiments in EE2 and EE3, respectively. Since these two environments are different from EE1, the RSSIbased localization models of the other methods are no longer suitable. As a result, other methods perform more poorly in the new environments. On the contrary, the proposed method already considers possible changes in the environment and preformed more robustly in different environments.

\section{B. Intruder Detection of the On-Demand Robot}

The experiment aimed to demonstrate the application of a WSN-based location aware system and mobile robot for intruder detection. Fig. 6 shows the system architecture of the experiment. Three intruder sensor modules were placed in the environment, including one microphone and two pyro sensors as well as a three-axis accelerometer. The sensing data from the intruder sensor modules were first collected and

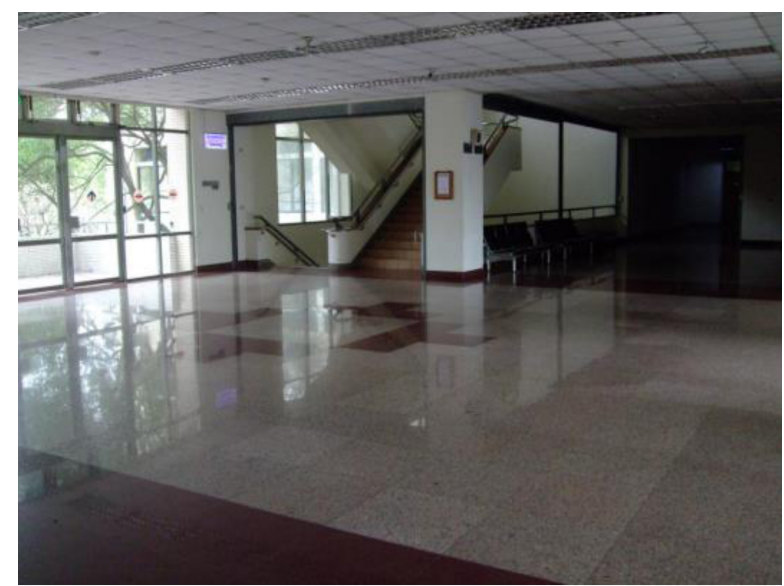

(a)
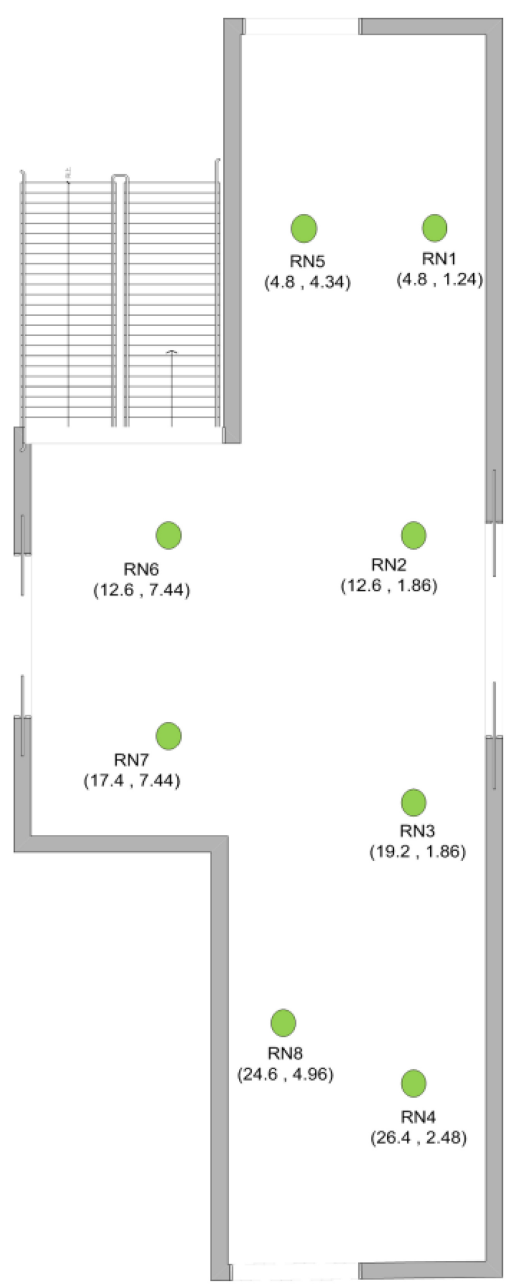

(b)

Fig. 12. (a) Experimental environment 3 (EE3). Lobby of the lab building. (b) Location of deployed ZigBee beacons in the experiment (filled circles).

processed on the modules independently. A location aware system was formed using sensor modules, and other ZigBee nodes deployed in the environment. The system can provide the locations of the nodes installed on the sensor modules and the robot. If an intruder is detected, the ID of the module and related sensor data will be transmitted to the mobile 
TABLE III

ESTIMATION ERROR COMPARISON: EE2

\begin{tabular}{c|ccc}
\hline \hline Method & $\begin{array}{c}\text { Average } \\
\text { Error }(\mathbf{m})\end{array}$ & $\begin{array}{c}\text { Max Error } \\
(\mathbf{m})\end{array}$ & $\begin{array}{c}\text { Standard } \\
\text { Deviation }\end{array}$ \\
\hline Min-Max & 2.46 & 3.2 & 1.14 \\
ROCRSSI & 2.76 & 3.1 & 1.22 \\
CC2431 & 1.82 & 3.5 & 1.5 \\
$\begin{array}{c}\text { Proposed } \\
\text { (2D Map) }\end{array}$ & 1.32 & 3 & 0.73 \\
$\begin{array}{c}\text { Proposed } \\
\text { (with Filtered Map) }\end{array}$ & 1.21 & 3.2 & 0.92 \\
\hline \hline
\end{tabular}

TABLE IV

ESTIMATION ERROR COMPARISON: EE3

\begin{tabular}{c|ccc}
\hline \hline Method & $\begin{array}{c}\text { Average Error } \\
(\mathbf{m})\end{array}$ & $\begin{array}{c}\text { Max Error } \\
(\mathbf{m})\end{array}$ & $\begin{array}{c}\text { Standard } \\
\text { Deviation }\end{array}$ \\
\hline Min-Max & 3.7 & 6.3 & 1.29 \\
ROCRSSI & 3.1 & 5.2 & 1.61 \\
CC2431 & 2.2 & 7.58 & 1.74 \\
$\begin{array}{c}\text { Proposed } \\
\text { (2D Map) }\end{array}$ & 1.5 & 4.2 & 0.89 \\
$\begin{array}{c}\text { Proposed } \\
\text { (with Filtered Map) }\end{array}$ & 1.36 & 3.21 & 0.73 \\
\hline \hline
\end{tabular}

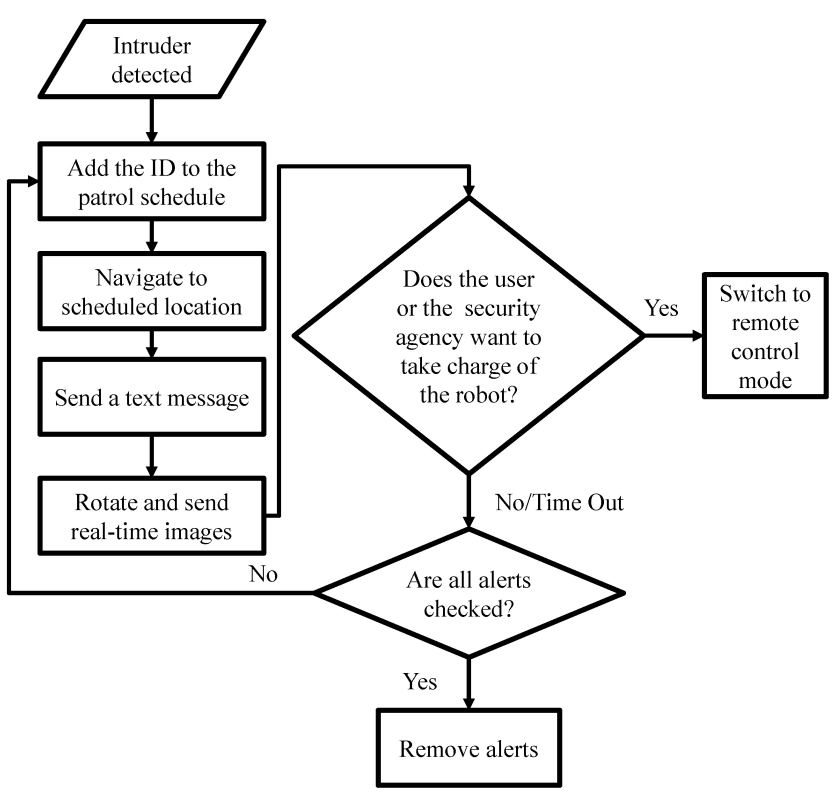

Fig. 13. Data flow of the ZigBee localization experiments.

robot via the WSN. The current position of the robot and the triggered module are also determined by the location aware system at the same time. Meanwhile, the mobile robot will receive the detection result from the WSN, navigate to each of the alarm locations, and take real-time images using the robot's webcam. The security agency and users can easily access both the detection result and real-time video transmitted by the robot on their portable devices via both WiFi and $3 \mathrm{G}$
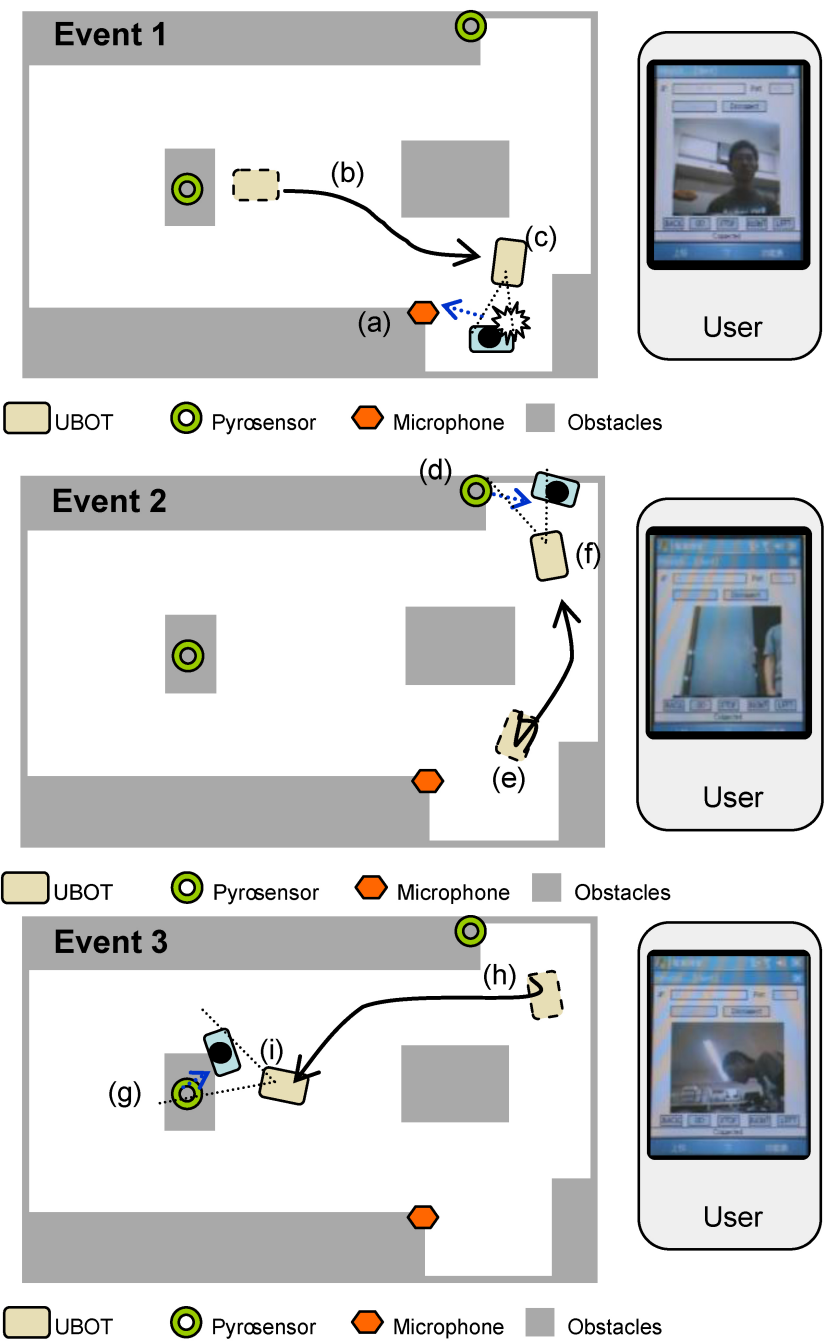

Fig. 14. Process and images acquired by the robot in the intruder detection experiment. (a) Person clapped his hands near the microphone. (b) Robot received the alert and moved to the person. (c) Robot took the person's image and sent it to the user. (d) Person walked into the room from the doorway. (e) Robot received the alert and moved to the person. (f) Robot took the person's image and sent it to the user. (g) Person (acting as a thief) intruded into the room. (h) Robot received the alert and moved to the person. (i) Robot took the image and sent it to the user.

networks. Users can also remotely control the robot to confirm the circumstances in detail.

Fig. 13 shows the execution flowchart of the intrusion detection system. Once a sensor module is triggered by the detection of a possible intruder, the ID of that module will be logged and added to the patrol schedule. Additional redundant triggers will then be ignored. Once the robot arrives at the alarm location, it will send both a short message and real-time images to the security agency and end users. The operators can then take control of the robot if necessary. Otherwise, the robot will move to the next alarm position. In the experiment, three different cases were tested in one script, including situations that mimicked a broken window by hand clapping (Event 1), a person entering the room (Event 2), and a thief sneaking into the room (Event 3), see Fig. 14. Fig. 15 illustrates the experiment using snapshots. Fig. 16 shows the actual trajectory of the robot during the experiment. During Event 1, a person clapped 


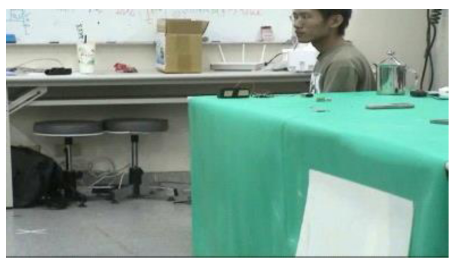

(a)

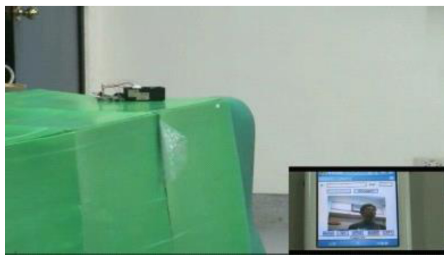

(d)

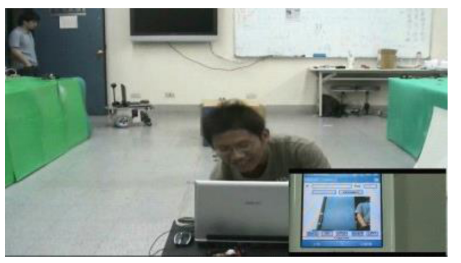

(g)

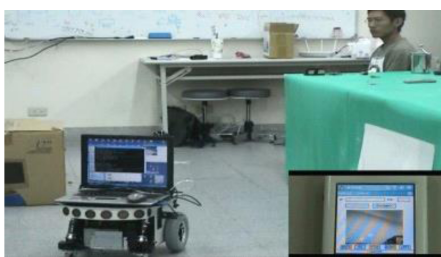

(b)

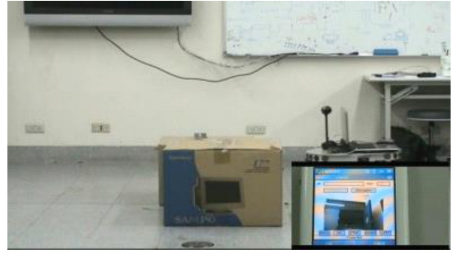

(e)

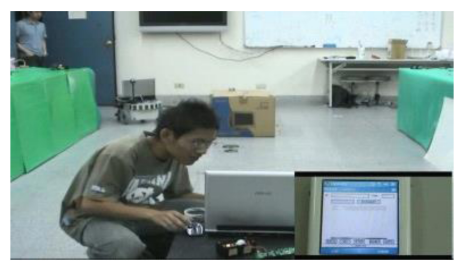

(h)

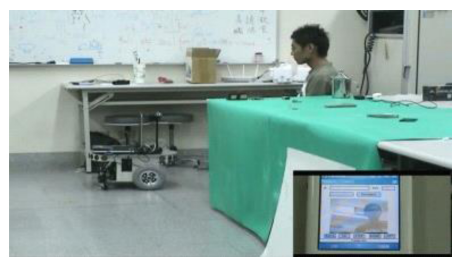

(c)

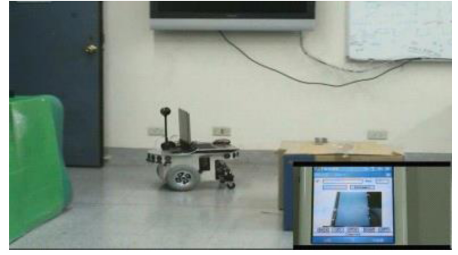

(f)

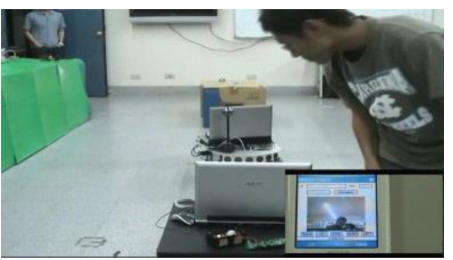

(i)

Fig. 15. Snapshots of the intruder detection experiment corresponding to Fig. 14. (a)-(c) correspond to Event 1, (d)-(f) correspond to Event 2, and (g)-(i) correspond to Event 3 in Fig. 14.

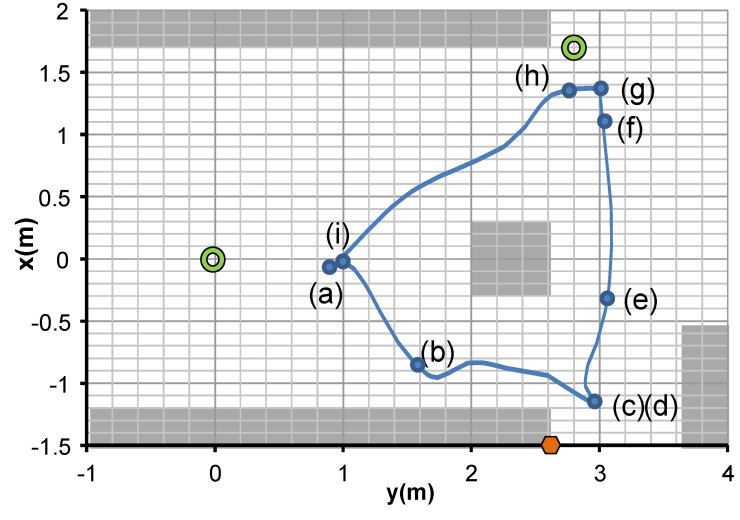

Fig. 16. Robot trajectory in the intruder detection experiment (a)-(i) Location of the robot in Fig. 15(a)-(i).

his hand and triggered the alarm, as shown in Figs. 14(a) and 15(a). The robot received the alarm signal and moved to where the corresponding surveillance location had been designated by the ZigBee sensor module [Figs. 14(b) and (c), 15(b) and (c), and 16(a)-(c)]. During Event 2, a person entered the room, which triggered the pyro sensor near the door. The robot reacted by moving toward the door [Figs. 14(d)-(f), 15(d)-(f), and 16(d)-(f)]. Finally, during Event 3, a person acting as a thief sneaked inside and wanted to steal a notebook. He triggered the pyro sensor and the robot once again approached the notebook and took pictures of the thief [Figs. 14(g)-(i), 15(g)-(i), and 16(g)-(i)]. Figs. 14 and 15 also show the images received by a remote user. Since these images were taken without using an algorithm and tracking mechanism, the system cannot be guaranteed to take any particular photos of the intruder. ${ }^{1}$

\section{CONCLUSION}

This paper presents a novel method for localizing a ZigBee node in a WSN. The probability-based approach takes the uncertainties of RSSI-based distance estimation into account. The method is independent of the sensor node distribution and environment configuration, and therefore can minimize the calibration burden in practical applications. The performance of the proposed algorithm has been evaluated by experiments in three different environments. The experimental results show that the average error of the implemented location aware system is $1.7 \mathrm{~m}$, despite a much higher range of uncertainties from raw RSSI values. This accuracy can effectively support service robotic applications, especially when properly combined with a vision system. For instance, the localization system can provide a possible area of a user calling the robot for service or when an emergency situation occurs. The robot can then find the alarm area and monitor it with its vision system. Use of a mobile robot to detect an intruder has been demonstrated. Three types of intrusion sensors have been installed and tested for the robotic patrolling system. This system can easily adopt other off-the-shelf sensor modules, while at the same time retaining compatibility with most existing security solutions. Our initial experiments show that the proposed system can effectively detect intruders and transmit their snapshots to portable devices at a remote site in real time. In the future,

${ }^{1} \mathrm{~A}$ video clip of the whole experiment can be found at http://isci.cn.nctu.edu.tw/Video/SMC2013. 
a distributed version will be designed and implemented. The position of the reference node will be saved and sent to the mobile node in the localization phase. Location-aware computation can be performed on the ZigBee module. Furthermore, the proposed method estimates the location from only a set of RSSI values without using previous RSSI values. Thus, it is also interesting to examine the performance of the target tracking with the proposed method.

\section{ACKNOWLEDGMENT}

The authors would like to thank K.-H. Yu and S.-H. Yang, Department of Electrical and Computer Engineering, National Chiao Tung University, Hsinchu, Taiwan for their support in performing the experiments.

\section{REFERENCES}

[1] R. C. Luo, T. Y. Lin, and K. L. Su, "Multisensor based security robot system for intelligent building," Robot. Autonom. Syst., vol. 57, no. 3, pp. 330-338, 2009.

[2] B. J. You, M. Hwangbo, S. O. Lee, S.R. Oh, Y. D. Kwon, and S. Lim, "Development of a home service robot 'ISSAC'," in Proc. IEEE/RSJ Int. Conf. Intell. Robots Syst., 2003, pp. 2630-2635.

[3] Y. G. Kim, H. K. Kim, S. H. Yoon, S. G. Lee, and K. D. Lee, "Home security robot based on sensor network," in Proc. SICE-ICASE Int. Joint Conf., 2006, pp. 5977-5982.

[4] R. Borja, J. R. de la Pinta, A. Álvarez, and J. M. Maestre, "Integration of service robots in the smart home by means of UPnP: A surveillance robot case study," Robot. Autonom. Syst., vol. 61, no. 2, pp. 153-160, 2013.

[5] K. T. Song, C. H. Lin, S. C. Tsai, S. C. Hung, Y. C. Liao, and C. C. Wu, "Development of robot-on-demand behaviors based-on visual tracking and wireless sensor network," in Proc. Int. Conf. Service Interactive Robots, 2011, pp. 261-266.

[6] C. Williams, Y. K. Cho, and J. H. Youn,"Wireless sensor-driven intelligent navigation method for mobile robot applications in construction," in Proc. ASCE Int. Workshop Comput. Civil Eng., 2007, pp. 493-498.

[7] K. Han, J. Lee, S. Na, and W. You "An ambient robot system based on sensor network: Concept and contents of ubiquitous robotic space," in Proc. Int. Conf. Mobile Ubiquitous Comput. Syst. Services Technol., 2007, pp. 155-159.

[8] G. Zanca, F. Zorzi, A. Zanella, and M. Zorzi, "Experimental comparison of RSSI-based localization algorithms for indoor wireless sensor networks," in Proc. Workshop Real-World Wireless Sensor Netw., 2008, pp. 1-5.

[9] C. Liu, T. Scott, K. Wu, and D. Hoffman, "Range-free sensor localisation with ring overlapping based on comparison of received signal strength indicator," Int. J. Sensor Netw., vol. 2, nos. 5-6, pp. 399-413, 2007.

[10] A. Savvides, H. Park, and M. B. Srivastava, "The n-hop multilateration primitive for node localization problems," Mobile Netw. Applicat., vol. 8 no. 4 , pp. 443-451, 2003

[11] K. Langendoen and N. Reijers,"Distributed localization in wireless sensor networks: A quantitative comparison," Comput. Netw., vol. 43, no. 4, pp. 499-518, 2003.

[12] F. Gustafsson and F. Gunnarsson, "Mobile positioning using wireless networks: Possibilities and fundamental limitations based on available wireless network measurements," IEEE Signal Process. Mag., vol. 22, no. 4, pp. 41-53, Jul. 2005

[13] G. I. Wassi, C. Despins, D. Grenier, and C. Nerguizian, "Indoor location using received signal strength of IEEE 802.11b Access Point," in Proc. Canad. Conf. Electr. Comput. Eng., 2005, pp. 1367-1370.

[14] K. T. Song, C. Y. Tsai, F. S. Huang, J. W. Hong, C. Y. Lin, C. W. Chen, and Z. S. Lin, "Development of the robot of living aid: RoLA," in Proc. IEEE Int. Conf. Autom. Logistics, Sep. 2008, pp. 443-448.

[15] T. Oliveira, M. Raju, and D. P. Agrawal, "Accurate distance estimation using fuzzy based combined RSSI/LQI values in an indoor scenario: Experimental verification," Netw. Protocols Algorithms J., vol. 4, no. 4, pp. 174-199, 2012.
[16] L. Gogolak, S. Pletl, and D. Kukolj, "Indoor fingerprint localization in WSN environment based on neural network," in Proc. IEEE 9th Int. Symp. Intell. Syst. Inform., Sep. 2011, pp. 293-296.

[17] S. H. Fang and T. N. Lin, "Indoor location system based on discriminantadaptive neural network in IEEE 802.11 environments," IEEE Trans. Neural Netw., vol. 19, no. 11, pp. 1973-1978, Nov. 2008.

[18] V. Ramadurai and M. L. Sichitiu, "Localization in wireless sensor networks: A probabilistic approach," in Proc. Int. Conf. Wireless Netw., Jun. 2003, pp. 275-281.

[19] J. Graefenstein and M. E. Bouzouraa, "Robust method for outdoor localization of a mobile robot using received signal strength in low power wireless networks," in Proc. IEEE Int. Conf. Robot. Autom., May 2008, pp. 33-38.

[20] R. Vaughan and J. Andersen, Channels, Propagation and Antennas for Mobile Communications, Institution of Electrical Engineers, London, U.K., 2003.

[21] H. Cho, M. Kang, J. Park, B. Park, and H. Kim, "Performance analysis of location estimation algorithm in ZigBee networks using received signal strength," in Proc. 21st Int. Conf. Adv. Inform. Netw. Applicat. Workshops, 2007, pp. 302-306.

[22] K. Lee, A. Oka, E. Pollakis, and L. H. Lampe, "A comparison between unscented kalman filtering and particle filtering for RSSI-based tracking," in Proc. 7th Workshop Positioning Navigation Commun., Mar. 2010, pp. 157-163.

[23] ZigBee Alliance. (2008). ZigBee Specification [Online]. Available: http://www.zigbee.org/

[24] C. H. Lin, S. H. Yang, H. T. Chen, and K. T. Song, "Mobile robot intruder detection based on a ZigBee sensor network," in Proc. IEEE Int. Conf. Syst. Man Cybern., Oct. 2008, pp. 2786-2791.

[25] S. H. Yang and K. T. Song, "An adaptive routing protocol for health monitoring with a sensor network and mobile robot," in Proc. IEEE IECON, 2010, pp. 2181-2186.

[26] K. T. Song and J. Y. Lin, "Behavior fusion of robot navigation using a fuzzy neural network," in Proc. IEEE Int. Conf. Syst., Man, Cybern., Oct. 2006, pp. 4910-4915.

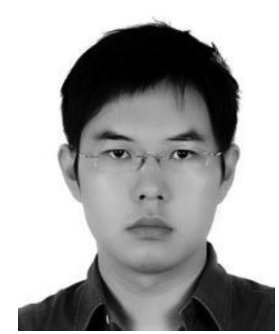

Chia-How Lin received the B.S. and M.S. degrees in electrical and control engineering from Nationa Chiao Tung University, Hsinchu, Taiwan, in 2001 and 2003, and is currently pursuing the Ph.D. degree in electrical control engineering at the same university.

His current research interests include multiagent systems, robot control systems, sensor networks, computer vision, and image-based robot navigation.

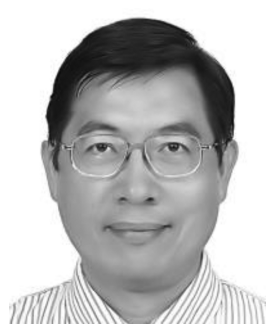

Kai-Tai Song (A'91-M'09) received the B.S. degree in power mechanical engineering from National Tsing Hua University, Hsinchu, Taiwan, in 1979, and the Ph.D. degree in mechanical engineering from the Katholieke Universiteit Leuven, Flanders, Belgium, in 1989.

Since 1989, he has been on the faculty and is currently a Professor with the Department of Electrical and Computer Engineering and the Institute of Electrical Control Engineering, National Chiao Tung University, Hsinchu, Taiwan. He served as the Chairman of the Society of IEEE Robotics and Automation, Taipei Chapter in 1999. His current research interests include mobile robots, image processing, visual tracking, mobile manipulation, embedded systems, and mechatronics.

Dr. Song was a recipient of the Excellent Award in Automatic Control Engineering from the Chinese Automatic Control Society (CACS), Taipei, Taiwan, in 2009. He was elected as a fellow of the CACS in 2010. He was also the recipient of the 2011 Engineering Paper Award from the Chinese Institute of Engineers and the Best Paper in Automation of IEEE ICAL in 2012. 